\title{
Puerperal Hematoma, Rare Postpartum Complication: A Case Report
}

\author{
Fatima Zahra Gounain*, N. Mamouni, S. Erraghay, C. Bouchikhi, A. Banani
}

Obstetrics Gynecology Department I in CHU Hassan Fez, Morocco

\begin{tabular}{ll}
\hline DOI: $10.36348 /$ sijog.2020.v03i12.005 & | Received: 07.11.2020 | Accepted: 19.11.2020 | Published: 24.12.2020
\end{tabular}

*Corresponding author: Fatima Zahra Gounain

\section{Abstract}

Post-partum hemorrhage is the leading cause of maternal mortality, ranging from 18 to $50 \%$ of deaths worldwide. The puerperal hematoma is an unusual cause (also called peri-genital thrombi) with a frequency of $1 / 1000$. They are formed in a paravaginal, paracervical or parametrial connective detachment, after vascular lesions. There are many favorable factors: primiparity, instrumental extraction, macrosomia, multiple pregnancies, vulvovaginalvarices, difficult hemostasis, coagulation anomalies, and prolonged dilation. The "active" treatment of puerperal hematoma is based on surgery and/or arterial embolization.

Keywords: Post-partum hemorrhage, paracervical, macrosomia, treatment.

Copyright (C) 2020 The Author(s): This is an open-access article distributed under the terms of the Creative Commons Attribution 4.0 International License (CC BY-NC 4.0) which permits unrestricted use, distribution, and reproduction in any medium for non-commercial use provided the original author and source are credited.

\section{INTRODUCTION}

Puerperal hematoma is a rare postpartum bleeding complication. In the CNGOF updates, dating from 1998, Jacquetin et al., consider that the term thrombus is inadequate and that it should be replaced by puerperal hematoma or perineal hematoma [1].

The thrombus corresponds to a detachment of the para-vaginal connective tissue in which the vascular wounds, linked to the detachment, have no spontaneous tendency to haemostasis: they indeed occur in loose adipose tissue and the hematoma can spread [2].

\section{PATIENT AND OBSERVATION}

Madam KS, 24 years old, G1P1, married, She was urgently referred to our department accompanied by a midwife for treatment of swelling of the right lips. The onset was marked by the immediate onset of profuse vaginal bleeding after childbirth, followed by very painful vulvar swelling (straight lips). What prompted the midwife who gave birth to the accompanied to the CHU for additional care after the conditioning of the parturient: establishment of a venous line with $5 \%$ glucose serum containing 25 units of syntocinon after the bleeding has stopped and before the episiotomy is repaired, the patient begins to develop extreme asthenia with skin-mucous membrane paleur and the appearance a few minutes later of a progressive and very painful swelling of the right labia majora. and she begins to develop shock with hypotension at 8 of the maximum out of 5 of the minimum noted that the patient was followed during the 3rd trimester of her pregnancy for preeclampsia and she was put on aldomet $500 \mathrm{mg}, 3$ tablets per day

On physical examination on admission to the gyneco-obstetrical emergency department in our training, the general condition was altered by extreme pallor and asthenia. Apyretic at 37.2 degrees Celsuis, with heart rate at 86 beats per minute hypotensive at $80 / 50$ millimeters of mercury and eupneic at 18 cycles per minute with conjunctival paleur. The rest of the somatic examination is unremarkable with the gynecological examination : good safety globe and on inspection of the external genitalia find bluish swelling of the right hemi vulva reaching the inguinal fold outwards, the gluteal fold behind, the mount of comers forward. Inside the mass pushed back the small lip and protruding into the vaginal wall from which a trickle of blood flowed through a tear involving the muscles and the mucous membrane (Figure). The skin was shiny, under strong tension and presented beaches of dermabrasions. Palpation was extremely painful and made it difficult to explore the mass without anesthesia. Its large diameter was anteroposterior and measured 20 centimeters while its small diameter, transverse, 
measured 10 centimeters. These dimensions increased quite rapidly as did the pain reported by the patient.

Examination of the limbs showed paleness of the extremities with no other abnormalities. We diagnosed immediate postpartum expansive vulvar hematoma complicated by severe anemia. For this we did an examination under local anesthesia with lidocaine. And we tried to empty the hematoma in order to achieve hemostasis with demand for blood by request for red blood cells (hemoglobin level at 6 grams / deciliter) after evacuation of the hematoma and an ice pack, we performed several hemostatic stitches using a number 1 vicryl thread (repair of the episiotomy site) and then compression for 12 hours with a vaginal tampon with good improvement on the clinical plan 12 hours later the patient was transferred to the ward for monitoring of blood pressure figures. Then the patient declared discharged after 72 hours of monitoring under no treatment with an appointment at the hospital of the day after one week with blood pressure figures and for monitoring of the hematoma site

\section{DISCUSSION}

Vulvovaginal thrombus is a rare but potentially serious obstetric complication, appearing in 1/700 deliveries for small hematomas, and 1/4000 for large ones [4].

It is due to a vascular rupture, often venous, which forms in the thickness of the vagina, after rolling of the wall and diffusion along the various fasciae. It is most often accompanied by a vulvar hematoma which can fuse below the levator ani muscle, distend the perineum and push back the rectum and anus. The hematoma can widen the vaginal wall considerably and also extend upward into the broad ligament base and retroperitoneum. There are four main types of genital hematoma [5]

Vulvar hematoma (Fig-1) here the bleeding is limited to the vulvar tissue and the tumor is easily diagnosed by sight. It is a hematoma the thickness of the labia majora, below the fascial plane of the perineum. It pushes the little lip back inside [6].

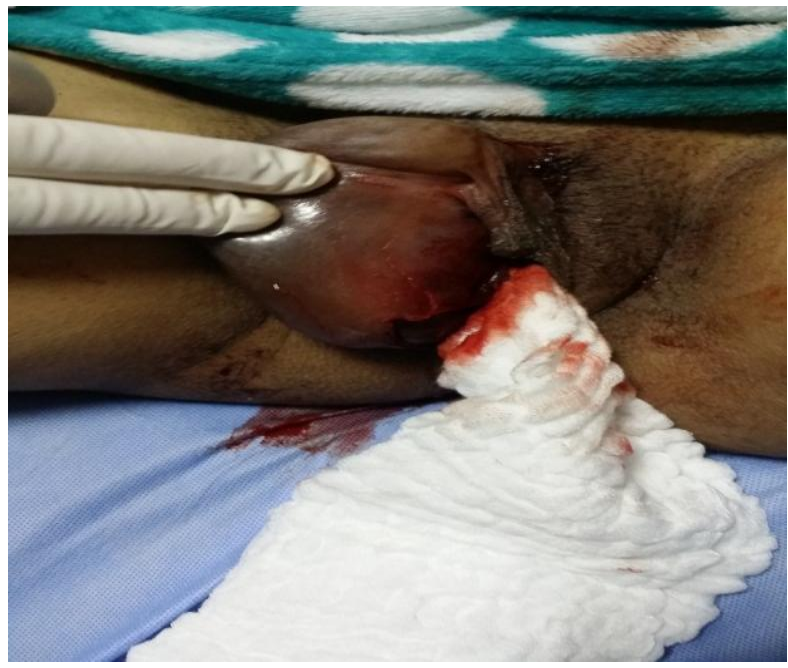

Fig-1: Expansive hematoma dissecting the right labia majora

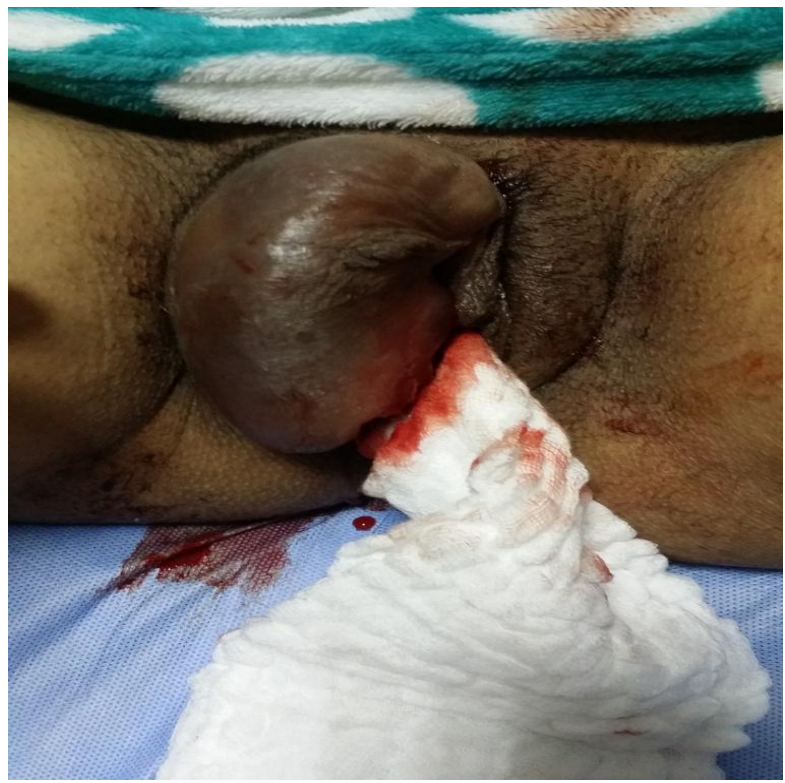

Fig-2

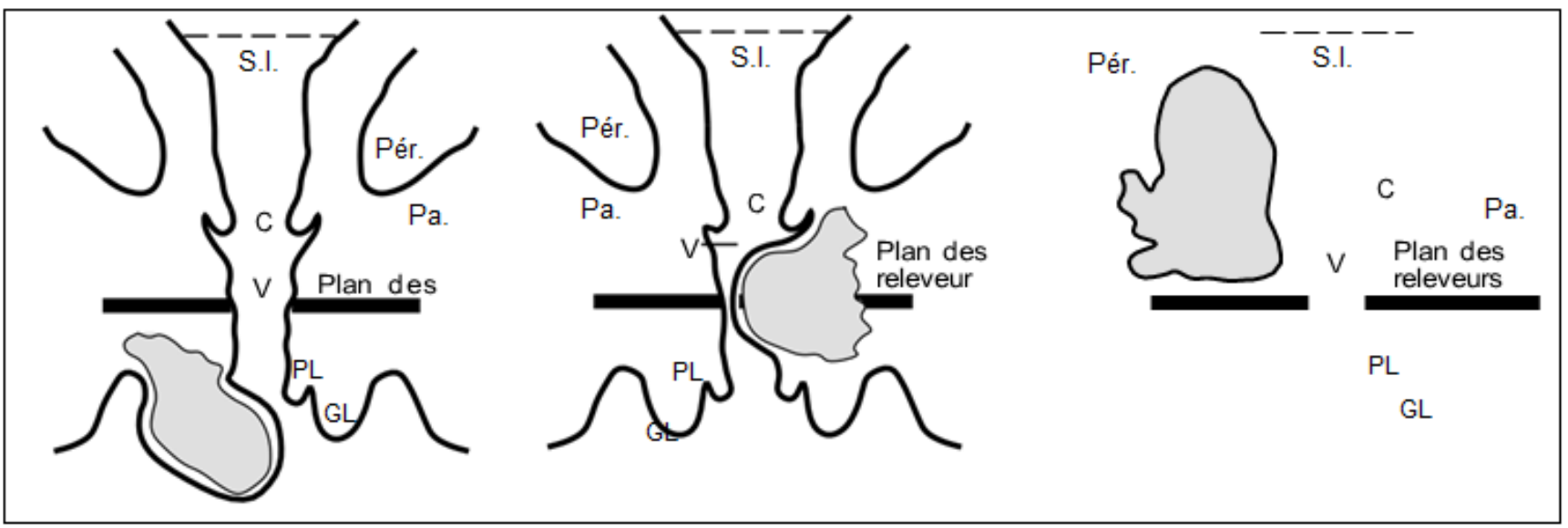

Fig-3: Pelvi-abdominal hematoma according 
Vulvovaginal hematoma. In this case, the hemoma involves, in addition to the vulva, the paravaginal tissues, the perineum and the ischial-rectal fossa. The collection is limited internally by the vagina, externally by the levator muscle and its aponeurosis.

The vaginal hematoma itself (Fig-2). It is limited to paravaginal tissue and is not diagnosed by simple vulvar inspection [6].

Pelvi-abdominal or supravaginal or subperitoneal hematoma (Fig-3). The bleeding occurred over the pelvic aponeuroses in the retroperitoneal or intraligamentous region. Pelvi-abdominal hematomas usually originate in a fissure in the lower segment [6].

The risk factors for HPV recognized in the literature are: primiparity, instrumental fetal extraction, pre-eclampsia, twin pregnancies, macrosomia and vulvovaginal varices [6].

In our patient, genital hematoma can be explained by preeclampsia and primiparity. As reported in the literature, the clinical picture in our patient was dominated by severe pain in the vagina, and unilateral swelling a few moments after childbirth and more specifically just after the completion of the episiotomy $[6,7]$.

Treatment should be started quickly because, in addition to the often excruciating pain, the maternal prognosis can be irreparably compromised. In fact, in many cases, no anatomical element opposes the progression of the collection, thereby leading to significant blood loss with consumption coagulopathy. Hemodynamic resuscitation is associated with the treatment of the lesion. A distinction must be made between the management of vulvovaginal col lections, which are the most frequent, from that of the infinitely rarer pelvic-abdominal collections [6].

The disease-modifying treatment is based on haemostasis and correction of haemodynamic disturbances and adjuvant measures include antibiotic prophylaxis, and pain relief [6-8].

Hemostasis can occur on its own by compressing the vascular lesion by the clot constituting the hematoma [6, 7]. Otherwise, a haemostatic procedure is required and consists first of all in a surgical exploration of the hematoma site (incision evacuation of the hematoma - hemostatic sutures in " $\mathrm{X}$ " drainage - wicking - vaginal tamponade on a probe indwelling urinary tract).

In our patient the proposed treatment will consist of an incision, hemostasis, tamponade after having performed a vascular filling, a blood count, Platelet and coagulation test under the guise of analgesia as well as antibiotic prophylaxis (amoxicillin- clavulanic acid). For the incision we took advantage of the incision of the episiotomy with enlargement on the inner edge of the hematoma on the vaginal side, after performing a vaginal tamponade, heavy and pressed tamponade with the placement of 4 wicks applying the vaginal wall against the lateral wall of the pelvis concerned for 24 hours. At the same time, a bladder catheter was placed. The course after 24 hours was marked by good clinical improvement, in particular the cessation of bleeding and pain. With normalization of blood pressure levels on monotherapy the patient declared discharged with an appointment after one week for blood pressure control and genital examination.

\section{CONCLUSION}

Postpartum genital thrombus or peri-genital puerperal hematoma is a rare but potentially very severe complication of childbirth.

Risk factors are well known, however, it can occur in the absence of risk factors. It is certain that it is desirable to carefully suture any tears and episiotomies in order to avoid the occurrence of a pelvic-genital hematoma by poor workmanship.

\section{REFERENCES}

1. Jacquetin, B., Boulleret, C., \& Fatton, B. (1998). Thrombus génitaux. CNGOF, Extrait des Mises à jour en Gynécologie et Obstétrique. 22è journées nationales: Paris, Tome XXII: 347-365.

2. Berthet, J., Colette, C., Maillet, R., \& Schaal, J. P. (1993). Mécanique et techniques obstétricales. Montpellier: Sauramps medical. 450-64

3. Bienstman-Pailleux, J., Huissoud, C., Dubernard, G., \& Rudigoz, R. C. (2009). Prise en charge des hématomes puerpéraux. Journal de gynécologie obstétrique et biologie de la reproduction, 38(3), 203-208.

4. Sadoul, G. (1986). Traumatismes des voies génitales basses et du périnée consécutifs à l'accouchement. Encycl Med Chir Paris, France, Urgences, 24213, A10.

5. Dumont, M., \& Công-Huê, L. (1966). Les hématomes génitaux du post-partum. Gynéc Obstét, 2:249-60.

6. Riethmuller, D., Pequegnot-Jeannin, C., Rabenja, C. A., Koeberle, P., Schaal, J. P., \& Maillet, R. (1997). A rare cause of postpartum hemorrhage: a genital thrombus. Journal de gynecologie, obstetrique et biologie de la reproduction, 26(2), 154.

7. Kehila, M., Khedher, S. B., Zeghal, D., \& Mahjoub, S. (2014). Prise en charge conservatrice des hématomes puerpéraux de gros volume: à propos de 3 cas. Pan African Medical Journal, 16(1):9.

8. Virgili, A., Bianchi, A., Mollica, G., \& Corazza, M. (2000). Serious hematoma of the vulva from a bicycle accident. A case report. The Journal of reproductive medicine, $45(8), 662$. 